\title{
Ombitasvir, paritaprevir, and ritonavir, with or without dasabuvir, plus ribavirin for patients with hepatitis $C$ virus genotype 1 or 4 infection with cirrhosis (ABACUS): a prospective observational study
}

\author{
Salvatore Petta, Marco Marzioni, Pierluigi Russo, Alessio Aghemo, Alfredo Alberti, Antonio Ascione, Andrea Antinori, Raffaele Bruno, \\ Savino Bruno, Antonio Chirianni, Giovanni Battista Gaeta, Edoardo G Giannini, Manuela Merli, Vincenzo Messina, Simona Montilla, \\ Carlo Federico Perno, Massimo Puoti, Giovanni Raimondo, Maria Rendina, Francesca Ceccherini Silberstein, Erica Villa, Anna Linda Zignego, \\ Luca Pani, Antonio Craxì, on behalf of the ABACUS study group* and the AIFA team*
}

\section{Summary}

Background We ran a compassionate use nationwide programme (ABACUS) to provide access to ombitasvir, paritaprevir, and ritonavir, with dasabuvir, plus ribavirin for hepatitis $\mathrm{C}$ virus (HCV) genotype 1 infection and ombitasvir, paritaprevir, and ritonavir, plus ribavirin for HCV genotype 4 infection in patients with cirrhosis at high risk of decompensation while approval of these regimens was pending in Italy.

Methods In this prospective observational study, we collected data from a compassionate use nationwide programme from March 17, 2014, to May 28, 2015. Patients with HCV genotype 1 infection and cirrhosis at high risk of decompensation were given coformulated ombitasvir $(25 \mathrm{mg})$, paritaprevir $(150 \mathrm{mg})$, and ritonavir (100 mg) once daily and dasabuvir $(250 \mathrm{mg}$ ) twice daily for 12 weeks (patients with HCV genotype $1 \mathrm{~b}$ infection) or 24 weeks (patients with HCV genotype 1a infection). Patients with HCV genotype 4 infection were given coformulated ombitasvir (25 mg), paritaprevir (150 mg), and ritonavir (100 mg) once per day for 24 weeks. All patients were given weight-based ribavirin. The primary efficacy endpoint was sustained virological response at week 12 after the end of treatment (SVR12), analysed by intention-to-treat. Univariate and multivariate logistic regression analyses were used to identify baseline characteristics associated with SVR12. Adverse events were recorded throughout the study.

Findings 728 (96\%) of 762 patients with cirrhosis who were given ombitasvir, paritaprevir, and ritonavir, with or without dasabuvir, plus ribavirin therapy for 12 or 24 weeks achieved SVR12. Logistic regression analyses identified that bilirubin concentrations of less than $2 \mathrm{mg} / \mathrm{dL}$ were associated with SVR12 (odds ratio [OR] $4 \cdot 76$ [95\% CI 1.83-12.3]; p=0 001). $166(23 \%)$ of 734 patients included in safety analyses had an adverse event. 25 (3\%) patients discontinued treatment because of adverse events. Asthenia was the most commonly reported adverse event, occurring in 36 (5\%) patients.

Interpretation Our findings suggest that the safety and effectiveness of ombitasvir, paritaprevir, and ritonavir, with or without dasabuvir, plus ribavirin in patients with HCV genotype 1 or 4 infection and cirrhosis at high risk of decompensation in a real-life setting are similar to those reported in clinical trials. The concordance with clinical trials provides reassurance that the reported efficacy of this treatment in clinical trials will translate to its use in routine clinical practice.

Funding Dipartimento Biomedico di Medicina Interna e Specialistica dell'Universita di Palermo.

\section{Introduction}

European guidelines on the treatment of chronic hepatitis $\mathrm{C}$ virus (HCV) infection regard patients with cirrhosis as a high-priority population for treatment. ${ }^{1}$ In Europe, the all-oral, three direct-acting antiviral (DAA) regimen of ombitasvir, paritaprevir, and the pharmacokinetic enhancer ritonavir, with dasabuvir, with or without ribavirin is approved for the treatment of $\mathrm{HCV}$ genotype 1 infection, whereas the two DAA regimen of ombitasvir, paritaprevir, and ritonavir, plus ribavirin is approved for the treatment of HCV genotype 4 infection. ${ }^{2,3}$

In previously untreated or treated patients with compensated cirrhosis, 114 (94\%) of 121 patients with genotype 1a infection had a sustained virological response at week 12 after the end of treatment (SVR12) with ombitasvir, paritaprevir, and ritonavir, with dasabuvir plus ribavirin for 24 weeks, and $60(100 \%)$ of 60 patients with genotype $1 \mathrm{~b}$ infection who were given a 12 week regimen of ombitasvir, paritaprevir, and ritonavir, with dasabuvir, without ribavirin achieved SVR12.,5 In previously untreated or treated patients with genotype 4 infection and compensated cirrhosis, 60 (98\%) of 61 patients who had 16 weeks of treatment and 57 (97\%) of 59 patients and 30 $(97 \%)$ of 31 patients, who had 12 weeks of treatment with the all-oral two-DAA regimen of ombitasvir, paritaprevir, and ritonavir, plus ribavirin achieved SVR12. ${ }^{6,7}$ The safety profiles of the ombitasvir, paritaprevir, and ritonavir, plus ribavirin regimens with or without dasabuvir in patients with compensated cirrhosis were similar to those in patients without cirrhosis. ${ }^{4-13}$
Lancet Gastroenterol Hepatol 2017; 2: 427-34

Published Online April 10, 2017 http://dx.doi.org/10.1016/ S2468-1253(17)30048-1

This online publication has been corrected. The corrected version first appeared at thelancet.com/gastrohep on May 5, 2017

*Members listed in the appendix Section of Gastroenterology and Hepatology, Biomedical Department of Internal and Specialized Medicine (DiBiMIS), University of Palermo, Palermo, Italy (S Petta MD, Prof A (raxì MD); Clinic of Gastroenterology and Hepatology, Università Politecnica delle Marche, Ancona, Italy (Prof M Marzioni MD); Italian Medicines Agency, Rome, Italy (P Russo MD, S Montilla PhD, Prof L Pani MD); L'Unità Operativa Gastroenterologia ed Epatologia, Fondazione IRCCS Ca' Granda Ospedale Maggiore Policlinico di Milano, Milan, Italy (A Aghemo MD); Department of Molecular Medicine, University of Padova, Padova, Italy (A Alberti MD); Centro per le malattie del Fegato, Ospedale Fatebenefratelli, Napoli, Italy (A Ascione MD); National Institute for Infectious Diseases, "Lazzaro Spallanzani" Istituto di Ricovero e Cura a Carattere Scientific (IRCCS), Rome, Italy (A Antinori MD), Dipartimento Malattie Infettive, Fondazione IRCCS Policlinico San Matteo Pavia Italia, Università degli studi di Pavia, Pavia, Italy (R Bruno MD); Humanitas University and Humanitas Research Hospital Rozzano, Milan, Italy 
(Prof S Bruno MD); UOC Infezioni sistemiche e

dell'immunodepresso, AO Ospedali dei Colli Napoli, Napoli, Italy (A Chirianni PhD); Infectious Diseases and Viral Hepatitis, Second University of Naples, Naples, Italy (Prof G B Gaeta MD); Gastroenterology Unit, Department of Internal Medicine, University of Genoa, Genoa, Italy (E G Giannini MD); Gastroenterology Department of Clinical Medicine, Sapienza University of Rome, Rome, Italy (Prof M Merli MD); Infectious Diseases Unit, AORN Sant'Anna e San Sebastiano, Caserta, Italy (V Messina MD); Department of Experimental Medicine and Surgery, University of Rome Tor Vergata, Rome, Italy (Prof C Federico Perno MD, Prof F Ceccherini Silberstein PhD); Department of Infectious Diseases, AO Niguarda Ca' Granda, Milan, Italy

(M Puoti MD); Division of Clinical and Molecular Hepatology, Department of Clinical and Experimental Medicine, University Hospital of Messina, Messina, Italy (G Raimondo MD); Gastroenterology and Digestive Endoscopy, University Hospital Policlinico Bari, Bari, Italy (M Rendina MD); Division of Gastroenterology, Azienda Ospedaliero, Universitaria Policlinico di Modena, Italy Università degli Studi di Modena e Reggio Emilia, Modena, Italy (Prof E Villa MD); and Interdepartmental Centre MASVE, Department of Experimental and Clinical Medicine, University of Florence, Florence, Italy (A L Zignego MD) Correspondence to: Dr Salvatore Petta, Section of Gastroenterology and Hepatology, Biomedical

Department of Internal and Specialized Medicine (DiBiMIS), University of Palermo, 90127 Palermo, Italy salvatore.petta@unipa.it

See Online for appendix
Research in context

\section{Evidence before this study}

We searched PubMed with search terms "ombitasvir", "paritaprevir", "dasabuvir", "genotype 1", "genotype 4", and "cirrhosis" for trials of the all-oral three direct-acting antiviral (DAA) regimen of ombitasvir, paritaprevir, and ritonavir, with dasabuvir, with or without ribavirin in patients with hepatitis $C$ virus (HCV) genotype 1 infection and the all-oral two-DAA regimen of ombitasvir, paritaprevir, and ritonavir, plus ribavirin in patients with $\mathrm{HCV}$ genotype 4 infection, published between Jan 1, 2014, and Dec 31, 2016. Only trials that included patients with compensated cirrhosis were selected. Three randomised, open-label trials and one single-arm, open-label trial were identified that met the criteria. In treatment-naive or treatment-experienced patients with compensated cirrhosis, treatment with ombitasvir, paritaprevir, and ritonavir, plus dasabuvir resulted in sustained virological response at week 12 after the end of treatment (SVR12) in $94 \%$ of patients with HCV genotype 1a infection who were given a 24 week regimen with ribavirin and $100 \%$ of patients with HCV genotype $1 \mathrm{~b}$ infection who were given a 12 week regimen without ribavirin.

Compassionate-use programmes for DAAs can provide access to drugs that have yet to be approved for high-priority patients with advanced, compensated cirrhosis who are at high risk of decompensation across a short timespan. Furthermore, these programmes provide additional early data on the effectiveness and safety of DAA regimens in general clinical practice and early experience of using new treatment regimens. With the support of the Italian Medicines Agency (Agenzia Italiana del Farmaco; AIFA), we did the AIFA-Based Associazione Italiana per lo Studio del Fegato (AISF) Compassionate Use Study (ABACUS) to provide access to ombitasvir, paritaprevir, and ritonavir, with dasabuvir, plus ribavirin for high-priority patients with genotype $1 \mathrm{HCV}$ infection and ombitasvir, paritaprevir, and ritonavir, plus ribavirin for high-priority patients with genotype 4 infection while approval of these regimens was pending in Italy. We report the efficacy and safety of ombitasvir, paritaprevir, and ritonavir, with or without dasabuvir, plus ribavirin in patients with cirrhosis who completed or prematurely discontinued treatment.

\section{Methods}

\section{Study design}

ABACUS was a prospective, longitudinal, observational study. ABACUS was not a clinical trial, but a compassionate use programme, so there was no planned number of patients or sites. The compassionate use programme was initiated by AbbVie (Campoverde, Italy) on March 17, 2014. Recruitment was stopped on May 28, 2015, when ombitasvir, paritaprevir, and ritonavir, with or without dasabuvir, plus ribavirin received marketing approval by AIFA. Patients were recruited at 176 sites in Italy via a network established by
In treatment-naive or treatment-experienced patients with HCV genotype 4 infection and compensated cirrhosis, the all-oral two-DAA regimen of ombitasvir, paritaprevir, and ritonavir, plus ribavirin resulted in $100 \%$ of patients achieving SVR12 after 16 weeks of treatment and $97 \%$ of patients achieving SVR12 after 12 weeks of treatment.

\section{Added value of this study}

This compassionate-use programme of ombitasvir, paritaprevir, and ritonavir, with or without dasabuvir, plus ribavirin for patients with HCV genotype 1 infection and ombitasvir, paritaprevir, and ritonavir, plus ribavirin for patients with HCV genotype 4 infection provides valuable data on the effectiveness and safety of these DAA regimens in general clinical practice.

Implications of all the available evidence

Our findings suggest that the safety and effectiveness of ombitasvir, paritaprevir, and ritonavir, with or without dasabuvir, plus ribavirin in patients with HCV genotype 1 or 4 infection and cirrhosis at high risk of decompensation in a real-world setting are similar to those reported in clinical trials.

AISF and AIFA. The data analysis cutoff was Feb 1, 2016. Patients were treated according to the European guidelines that were in use at the time of the study. Briefly, patients with HCV genotype 1 infection who had cirrhosis were given coformulated ombitasvir $(25 \mathrm{mg}$ ), paritaprevir $(150 \mathrm{mg})$, and ritonavir $(100 \mathrm{mg})$ once daily and dasabuvir $(250 \mathrm{mg}$ ) twice daily for 12 weeks (patients with genotype $1 \mathrm{~b}$ HCV infection) or 24 weeks (patients with genotype 1a HCV infection). Patients with genotype 4 infection who had cirrhosis were given coformulated ombitasvir (25 mg), paritaprevir $(150 \mathrm{mg})$, and ritonavir $(100 \mathrm{mg}$ ) for 24 weeks (appendix p 4). Ombitasvir, paritaprevir, ritonavir, and dasabuvir were provided by AbbVie. All patients were given weight-based doses of ribavirin $(1000 \mathrm{mg}$ for patients $<75 \mathrm{~kg}$, or $1200 \mathrm{mg}$ for patients $\geq 75 \mathrm{~kg}$ ) as recommended by the drug label. ${ }^{14}$ Blood transfusions and use of erythropoietin were permitted if deemed medically necessary.

The study was done in accordance with the International Conference on Harmonisation guidelines, applicable regulations at each study site, and the principles of the Declaration of Helsinki. Local ethics approval was obtained at each site.

\section{Patients}

The ABACUS compassionate-use programme provided treatment access for patients with HCV genotype 1 or 4 infection who had compensated cirrhosis and were at high risk of decompensation. High risk of decompensation was defined as clinical features suggestive of rapidly progressive fibrosis or impending decompensation (eg, a rapid decline in platelets, albumin concentration, or both) with previous signs or history of 
decompensation, or with rapid progression from histologically or clinically proven chronic HCV infection without cirrhosis to clinically diagnosed cirrhosis. All patients had Child-Pugh class A cirrhosis at the time of enrolment, but those with a history of decompensation were not excluded, and a change in Child-Pugh class could occur between enrolment and start of treatment. Diagnosis of cirrhosis at baseline was made by meeting at least one of the following criteria: histology from a liver biopsy, a FibroScan (Echosens, Paris, France) result of more than $14.6 \mathrm{kPa}$, a platelet count of less than 100000 cells per $\mu \mathrm{L}$, or the presence of oesophageal varices. Patients could be treatment naive (defined as never having had HCV treatment), or treatment experienced (defined as having previous treatment with interferon-based therapy, with or without ribavirin, and being a non-responder or a relapser, or interferonintolerant).

All patients in the study provided written informed consent before any study-specific procedures were carried out.

\section{Efficacy and safety assessments}

Efficacy and safety assessments were at the discretion of the investigators because this was a compassionate use programme and not a clinical trial. HCV RNA testing was done at the local laboratory at each site. However, investigators were requested to do laboratory assessments, including international normalised ratio (INR), albumin concentration, bilirubin concentration, alanine aminotransferase (ALT) concentration, and haemoglobin concentration, at baseline, at treatment weeks 2 and 4, every 4 weeks during treatment thereafter, and at week 12 after treatment. We assessed HCV RNA concentration at baseline, week 4 of treatment, end of treatment, and week 12 after treatment. We recorded data on adverse events throughout the study. Patient visits and follow-up, as well as laboratory testing, were done according to the sites' local standards. Laboratory and safety data were reported on an ongoing basis on a spreadsheet to the principal investigator. All clinicians were invited to contribute to data collection, but not all clinicians contributed.

\section{Outcomes}

The primary efficacy endpoint was the percentage of patients achieving an HCV RNA concentration of less than $25 \mathrm{IU} / \mathrm{mL} 12$ weeks after the end of therapyie, SVR12-by intention to treat. Secondary endpoints were the percentage of patients with virological breakthrough during treatment or relapse after treatment; effect of antiviral therapy on markers of liver function, such as INR, albumin concentration, and bilirubin concentration; safety events consistent with hepatic decompensation; and deaths during the study. We defined virological breakthrough during treatment as a confirmed HCV RNA concentration of $25 \mathrm{IU} / \mathrm{mL}$ or higher after achieving an HCV RNA concentration of lower than $25 \mathrm{IU} / \mathrm{mL}$. We defined virological relapse as a confirmed HCV RNA concentration of $25 \mathrm{IU} / \mathrm{mL}$ or higher between the end of treatment and 12 weeks after the last dose of study drug in patients who had an HCV RNA concentration of lower than $25 \mathrm{IU} / \mathrm{mL}$ at the final visit during the treatment period.

\section{Statistical analysis}

We presented categorical variables as percentages, and continuous variables as mean (SD). We compared patients who achieved SVR12 with those who did not, and those who had a serious adverse event (including death, liver decompensation, infection, haemoglobin concentration $<8 \mathrm{~g} / \mathrm{dL}$, or bilirubin concentration $>10 \mathrm{~g} / \mathrm{dL}$ ) with those who did not using Pearson's $\chi^{2}$ test for categorical variables and Student's $t$ test for continuous data. We used univariate and multivariate logistic regression models to assess baseline characteristics independently associated with SVR12 or serious adverse events. We modelled SVR12 and serious adverse events as the dependent variables. We regarded baseline characteristics associated with SVR12 or serious adverse events in univariate analyses $(\mathrm{p} \leq 0 \cdot 1)$ as independent variables in the multivariate logistic regression models. We tested changes in biochemical parameters over time by means of a paired two-sample $t$ test for comparisons that involved only two timepoints and by a linear mixed-effect regression model over time (with an intercept random effect for each patient) for comparisons that involved more than two timepoints. All analyses were done using IBM SPSS (version 18). We did efficacy analyses for patients with cirrhosis in the intention-to-treat (ITT) population (defined as all enrolled patients who were given at least one dose of study drug), and the per-protocol population (defined as all enrolled patients who completed treatment or discontinued treatment due to virological breakthrough).

\section{Role of the funding source}

The funder of the study had no role in study design, data collection, data analysis, data interpretation, or writing of the report. All authors had full access to all the data in the study and had final responsibility for the decision to submit for publication.

\section{Results}

Patients were enrolled in the study between March 17, 2014, and May 28, 2015. 934 patients with cirrhosis were given ombitasvir, paritaprevir, and ritonavir, with or without dasabuvir, plus ribavirin. 762 patients were included in the ITT population, 728 were included in the per-protocol population, and 734 were included in the safety population (figure; appendix p 5). Of the 172 patients who were not included in the ITT analysis, 128 were still on treatment or in the follow-up period before the week 12 follow-up visit at the time of the analysis, and clinicians chose not to provide 
data for analysis and dissemination for the remaining 44 patients. Baseline characteristics of patients with cirrhosis included in the study compared with those not included in the analysis were not substantially different. No patients were lost to follow-up. Baseline demographics and disease characteristics are presented in table 1 . Previous decompensation was recorded in seven (18\%) of 39 patients with Child-Pugh class B cirrhosis. The mean age of patients with Child-Pugh class B cirrhosis was 62.8 years (SD 9.4) and 24 (62\%) patients were men. Patients with Child-Pugh class B cirrhosis at baseline were treated at the discretion of the site investigator. All patients with Child-Pugh A were treated. All 39 patients with ChildPugh class B cirrhosis were included in the ITT population, and 33 of these patients were included in the per-protocol population.

$728(96 \%)$ of 762 patients in the ITT population who were given ombitasvir, paritaprevir, and ritonavir, with or without dasabuvir, plus ribavirin therapy for 12 or 24 weeks achieved SVR12 (table 2). The proportions of patients achieving SVR12 were similar in centres treating fewer than ten patients compared with centres treating ten or more patients (data not shown). SVR12 rates in treatment history and baseline HCV RNA concentration subgroups did not differ from the overall proportion of patients achieving SVR12 (table 2). The proportion of patients achieving SVR12 did not differ substantially between treatment-naive and treatmentexperienced patients in $\mathrm{HCV}$ genotype subgroups

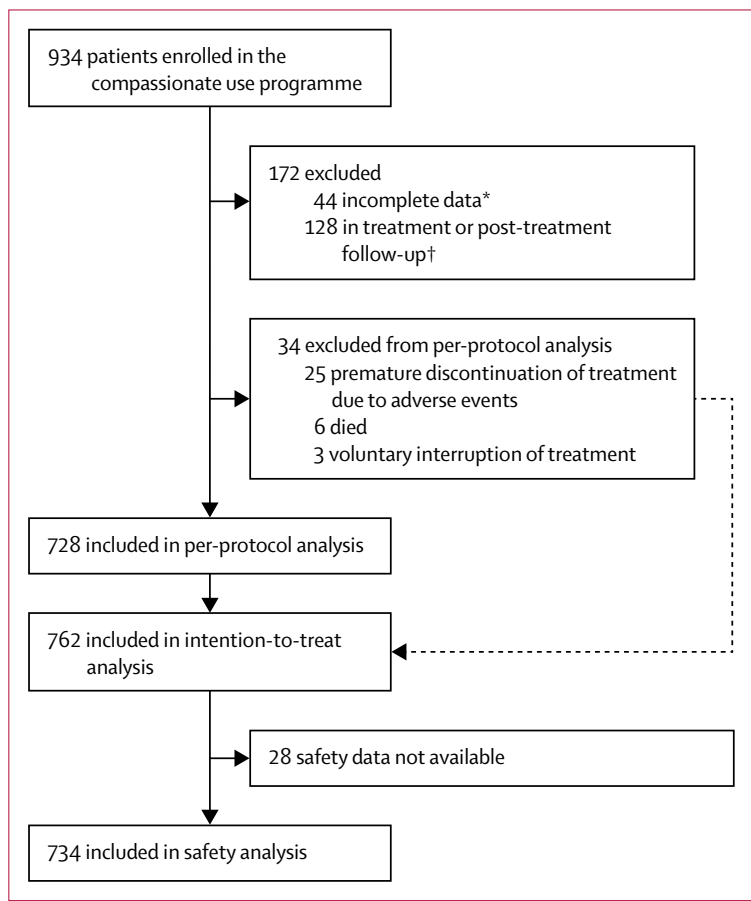

Figure: Study populations

*Data incomplete: treating physician chose not to provide data for analysis and dissemination. †Patients still on treatment, or in follow-up period before week 12 follow-up visit at the time of the analysis. (appendix p 6). There were significant differences in the proportion of patients achieving SVR12 by HCV genotype 1 subtype in the per-protocol population (genotype 1a 137 [95\%] of 144 patients vs genotype $1 \mathrm{~b}$ 545 [98\%] of 554 patients; $p=0 \cdot 02$ ). There was no significant difference in the ITT population (table 2). The proportion of patients achieving SVR12 did not differ substantially by age, sex, or body-mass index (appendix p 7), renal function (appendix p 8), or by baseline FibroScan result (appendix p 9) in the ITT population. Analyses of SVR by age, sex, body-mass index, renal function, and baseline FibroScan score were not done in the per-protocol population. Fewer patients with Child-Pugh class B cirrhosis achieved SVR12 than patients with Child-Pugh A cirrhosis $(\mathrm{p}<0 \cdot 0001$; appendix $\mathrm{p} \mathrm{10)}$; there was no significant difference in SVR rate by MELD score. The proportion of patients achieving SVR12 was lower in patients with markers of more severe liver disease $(\mathrm{p}=0.01$ for platelet count $<100000$ cells per $\mu \mathrm{L} ; \mathrm{p}=0.01$ for albumin $<3.5 \mathrm{~g} / \mathrm{dL}$; and $\mathrm{p}=0 \cdot 001$ for bilirubin $\geq 2 \mathrm{mg} / \mathrm{dL}$; appendix pp 10-11) by ITT. The proportion of patients achieving SVR12 who had HCV RNA concentrations of $25 \mathrm{IU} / \mathrm{mL}$ or lower at week 4 was significantly higher than those with HCV RNA concentrations higher than $25 \mathrm{IU} / \mathrm{mL}$ at week 4 $(\mathrm{p}=0.002$; appendix $\mathrm{p} 12)$. This was predominantly driven by the subgroup of patients with HCV genotype $1 b$ infection who were given ombitasvir, paritaprevir, ritonavir, with dasabuvir plus ribavirin for 12 weeks. Ribavirin dose had no effect on the proportion of patients achieving SVR12 (appendix p 13).

In the per-protocol population, a lower proportion of patients achieving SVR12 was observed in patients with bilirubin concentration of $2 \mathrm{mg} / \mathrm{dL}$ or higher $(\mathrm{p}=0.003$; appendix $\mathrm{p} 11)$, but no significant differences in SVR12 were recorded by presence of varices (appendix p 10) or other markers of severe liver disease (appendix p 11).

In the multivariate logistic regression analysis, the only factor with a significant association with SVR12 in the ITT population was a baseline bilirubin concentration of less than $2 \mathrm{mg} / \mathrm{dL}$ (odds ratio [OR] 4.76 [95\% CI $1 \cdot 83-12 \cdot 3$ ]; $p=0 \cdot 001)$. In the per-protocol population, baseline factors associated with achieving SVR12 were genotype $1 \mathrm{~b}$ infection (OR 2.97 [95\% CI 1.08-8.22]; $\mathrm{p}=0.03)$ and bilirubin concentration of less than $2 \mathrm{mg} / \mathrm{dL}$ (4.88 [1.62-14.6]; $\mathrm{p}=0.005)$. In patients with HCV genotype $1 \mathrm{~b}$ infection, baseline bilirubin concentration of lower than $2 \mathrm{mg} / \mathrm{dL}$ was independently associated with SVR12 (OR 5.09 [95\% CI 1.74-14.8]; $\mathrm{p}=0.003$ ). In patients with HCV genotype 1a infection, SVR12 was marginally, though not significantly, associated with baseline bilirubin concentration of lower than $2 \mathrm{mg} / \mathrm{dL}$ $(6 \cdot 70$ [0.95-46.9]; $\mathrm{p}=0 \cdot 05)$ and baseline serum albumin concentration of $3.5 \mathrm{~g} / \mathrm{dL}$ or higher $(6 \cdot 98$ [1.21-40.2]; $\mathrm{p}=0 \cdot 03)$. There were too few patients with genotype 4 infection for subgroup analysis. 


\begin{tabular}{|c|c|}
\hline & $\begin{array}{l}\text { ITT population } \\
(n=762)\end{array}$ \\
\hline Men & $437(57 \%)$ \\
\hline Age (years) & $59 \cdot 2(10 \cdot 4)$ \\
\hline \multicolumn{2}{|l|}{ Body-mass index } \\
\hline$\geq 30 \mathrm{~kg} / \mathrm{m}^{2}$ & $64(8 \%)$ \\
\hline$<30 \mathrm{~kg} / \mathrm{m}^{2}$ & $561(74 \%)$ \\
\hline Unknown & $137(18 \%)$ \\
\hline \multicolumn{2}{|l|}{ Comorbidities } \\
\hline Lymphoproliferative disorder & $20(3 \%)$ \\
\hline Cardiovascular disease & $29(4 \%)$ \\
\hline Arterial hypertension & $101(13 \%)$ \\
\hline Diabetes & $95(12 \%)$ \\
\hline Thyroid dysfunction & $25(3 \%)$ \\
\hline Psychiatric disorder & $27(4 \%)$ \\
\hline Creatinine $(\mathrm{mg} / \mathrm{dL})$ & $0.8(0.5)$ \\
\hline Creatinine clearance $(\mathrm{mL} / \mathrm{min})$ & $92 \cdot 4(16.9)$ \\
\hline \multicolumn{2}{|l|}{ Creatinine clearance $\left(\mathrm{mL} / \mathrm{min}\right.$ per $\left.1.73 \mathrm{~m}^{2}\right)$} \\
\hline$\geq 90$ & $501(66 \%)$ \\
\hline $60-89$ & $226(30 \%)$ \\
\hline $30-59$ & $31(4 \%)$ \\
\hline$<30$ & $4(1 \%)$ \\
\hline Alanine aminotransferase (IU/L) & $89.0(61.9)$ \\
\hline Total bilirubin (mg/dL) & $1 \cdot 1(0 \cdot 6)$ \\
\hline International normalised ratio & $1.0(0.2)$ \\
\hline Albumin (g/dL) & $3.9(0.4)$ \\
\hline Platelets ( $10^{\circ}$ cells per $\mathrm{L}$ ) & $129(74)$ \\
\hline Child-Pugh class B & $39(5 \%)$ \\
\hline MELD score $\geq 10$ & $185(24 \%)$ \\
\hline \multicolumn{2}{|l|}{ Oesophageal varices } \\
\hline Absent & $445(58 \%)$ \\
\hline Present & $210(28 \%)$ \\
\hline Unknown & $107(14 \%)$ \\
\hline \multicolumn{2}{|l|}{ HCV genotype } \\
\hline 1 (subtype unknown) $\dagger$ & $16(2 \%)$ \\
\hline $1 \mathrm{a}$ & $154(20 \%)$ \\
\hline $1 b$ & $575(75 \%)$ \\
\hline 4 & $17(2 \%)$ \\
\hline \multicolumn{2}{|l|}{ Baseline HCV RNA concentration } \\
\hline$>1000000 \mathrm{IU} / \mathrm{mL}$ & $385(51 \%)$ \\
\hline$<1000000 \mathrm{IU} / \mathrm{mL}$ & $348(46 \%)$ \\
\hline Unknown & $29(4 \%)$ \\
\hline Treatment naive & $235(31 \%)$ \\
\hline History of previous liver decompensation & $44(6 \%)$ \\
\hline \multicolumn{2}{|c|}{$\begin{array}{l}\text { Data are }(\%) \text { or mean (SD). ITT=intention to treat. HCV=hepatitis C virus. } \\
\text { *Estimated glomerular filtration rate calculated by the Chronic Kidney Diseas } \\
\text { Epidemiology Collaboration equation. }+ \text { Patients with unknown subtypes. }\end{array}$} \\
\hline
\end{tabular}

In the ITT population, fewer patients achieved SVR12 who had bilirubin concentrations of $2 \mathrm{mg} / \mathrm{dL}$ or higher and albumin concentrations of lower than $3.5 \mathrm{~g} / \mathrm{dL}$ compared with those with other combinations of bilirubin and albumin values (appendix $\mathrm{p} \mathrm{14}$ ). In the

\begin{tabular}{|lll}
\hline & $\begin{array}{l}\text { Proportion of patients } \\
\text { who achieved SVR12 }\end{array}$ & p value \\
\hline All & $728 / 762(96 \%)$ &.. \\
\hline $\begin{array}{l}\text { Treatment history } \\
\text { Treatment-naive }\end{array}$ & $222 / 235(94 \%)$ & 0.33 \\
Had pegylated interferon & $506 / 527(96 \%)$ &.. \\
treatment & & \\
Baseline HCV RNA concentration & \\
$<6$ log IU/mL & $336 / 348(97 \%)$ & 0.40 \\
$\geq 6$ log IU/mL & $367 / 385(95 \%)$ &.. \\
HCV genotype & & \\
1 & $712 / 745(96 \%)$ &.. \\
$1 \mathrm{a}$ & $143 / 154(93 \%)$ & $0.06(\mathrm{vs} 1 \mathrm{~b})$ \\
$1 \mathrm{~b}$ & $554 / 575(96 \%)$ &.. \\
4 & $16 / 17(94 \%)$ &.. \\
\hline
\end{tabular}

Data are $\mathrm{n} / \mathrm{N}(\%) . \mathrm{HCV}=$ hepatitis $\mathrm{C}$ virus. SVR12=sustained virological response at week 12 after the end of treatment. ITT=intention to treat.

Table 2: SVR12 in all patients with cirrhosis and by treatment history, baseline HCV RNA concentration, and HCV subtype (ITT population)

per-protocol population, the proportion of patients achieving SVR12 was lower in patients with HCV genotype 1a infection and bilirubin concentrations of $2 \mathrm{mg} / \mathrm{dL}$ or higher compared with those with HCV genotype 1a infection and bilirubin concentrations lower than $2 \mathrm{mg} / \mathrm{dL}$ (appendix p 15).

Of patients who achieved SVR12, markers of liver function significantly improved by week 12 after treatment compared with baseline (appendix p 16). There was no significant difference in INR $(\mathrm{p}=0 \cdot 34)$. Virological breakthrough occurred in four (1\%) of 762 patients in the ITT population, whereas virological relapse occurred in $14(2 \%)$ of 762 patients. None of the patients who did not achieve SVR12 had liver decompensation up to 12 weeks after treatment. Most patients with Child-Pugh class B cirrhosis at baseline who achieved SVR12 had an improvement in Child-Pugh score, MELD score, or both (appendix p 17).

ALT concentrations rapidly decreased to normal levels with ombitasvir, paritaprevir, and ritonavir, with or without dasabuvir, plus ribavirin for 12 weeks, and remained at normal levels 12 weeks after treatment (appendix p 18). Bilirubin concentrations were raised at week 2 of treatment, but gradually decreased thereafter, and reached normal levels at week 12 after treatment (appendix p 18). Haemoglobin concentrations decreased with start of treatment, but plateaued at week 4 , and returned to baseline values at week 12 after treatment (appendix p 19). Similar results were obtained with ombitasvir, paritaprevir, and ritonavir, with or without dasabuvir, plus ribavirin for 24 weeks (appendix p 20). Estimated creatinine clearance decreased during treatment with ombitasvir, paritaprevir, and ritonavir, with or without dasabuvir, plus ribavirin and remained lower than baseline at week 12 after treatment 


\begin{tabular}{|c|c|c|c|}
\hline & $\begin{array}{l}\text { Child-Pugh A } \\
(n=696)\end{array}$ & $\begin{array}{l}\text { Child-Pugh B } \\
(n=38)\end{array}$ & $\begin{array}{l}\text { All patients } \\
(\mathrm{n}=734)\end{array}$ \\
\hline \multicolumn{4}{|l|}{ Safety summary } \\
\hline Any adverse event & $149(21 \%)$ & $17(45 \%)$ & $166(23 \%)$ \\
\hline $\begin{array}{l}\text { Adverse event leading to } \\
\text { discontinuation of study } \\
\text { drugs }\end{array}$ & $21(3 \%)$ & $4(11 \%)$ & $25(3 \%)^{*}$ \\
\hline Death & $4(1 \%)$ & $2(5 \%)$ & $6(1 \%) \dagger$ \\
\hline \multicolumn{4}{|c|}{ Most common adverse events and adverse events of special interest } \\
\hline Asthenia & $34(5 \%)$ & $2(5 \%)$ & $36(5 \%)$ \\
\hline $\begin{array}{l}\text { Haemoglobin } \\
\text { concentration }<8 \mathrm{~g} / \mathrm{dL}\end{array}$ & $17(2 \%)$ & $1(3 \%)$ & $18(2 \%)$ \\
\hline Infection & $8(1 \%)$ & $3(8 \%)$ & $11(1 \%)$ \\
\hline $\begin{array}{l}\text { Total bilirubin } \\
\text { concentration }>10 \mathrm{mg} / \mathrm{dL}\end{array}$ & $5(1 \%)$ & $3(8 \%)$ & $8(1 \%)$ \\
\hline Cardiovascular & $7(1 \%)$ & 0 & $7(1 \%)$ \\
\hline Nausea & $6(1 \%)$ & 0 & $6(1 \%)$ \\
\hline $\begin{array}{l}\text { Hepatic } \\
\text { decompensation } \neq\end{array}$ & $3(<1 \%)$ & $1(3 \%)$ & $4(1 \%)$ \\
\hline Renal failure & $2(<1 \%)$ & 0 & $2(<1 \%)$ \\
\hline \multicolumn{4}{|c|}{$\begin{array}{l}\text { Data are } \mathrm{n}(\%) \text {. Most common adverse events were those that occurred in } 5 \% \text { of } \\
\text { patients or more in either group, while adverse events of special interest were } \\
\text { anaemia (haemoglobin concentration }<8 \mathrm{~g} / \mathrm{dL} \text { ), hyperbilirubinaemia (total } \\
\text { bilirubin }>10 \mathrm{mg} / \mathrm{dL} \text { ), gastrointestinal adverse events, cardiovascular adverse } \\
\text { events, renal adverse events, and those adverse events that were related to liver } \\
\text { function or infections. *Included four cases of anaemia, three cases of infection, } \\
\text { three cases of hyperbilirubinaemia, two cases of hepatic decompensation, and } \\
\text { one case of hepatocellular carcinoma. †Two deaths were due to multiple organ } \\
\text { failure (one as a result of cholecystitis leading to sepsis; one as a result of acute } \\
\text { kidney injury following resection of hepatocellular carcinoma), one was due to } \\
\text { hepatopulmonary syndrome as a result of pneumonia, one was due to a car } \\
\text { accident, one was due to lymphoma, and one sudden death of unknown cause. } \\
\text { łlncluded variceal haemorrhage. }\end{array}$} \\
\hline
\end{tabular}

(appendix p 22). There were no changes in blood glucose concentration during treatment and 12 weeks after treatment (appendix p 24).

$166(23 \%)$ of 734 patients had an adverse event. Rates of individual adverse events were low (table 3). Asthenia was the most commonly reported adverse event, occurring in $36(5 \%)$ of patients. Severe anaemia ( $<8 \mathrm{~g} / \mathrm{dL}$ haemoglobin) occurred in $18(2 \%)$ patients. Blood transfusions were given to $14(2 \%)$ patients and erythropoietin was given to 27 (4\%) patients. Occurrences of hepatic decompensation (four [1\%] of 734 patients) and renal failure (two $[<1 \%]$ of 734 patients) were low. $25(3 \%)$ of 734 patients discontinued study drugs due to an adverse event. There were six deaths during the study (table 3). Multivariate logistic regression analysis showed that bilirubin concentration of $2 \mathrm{~g} / \mathrm{dL}$ or higher was significantly associated with serious adverse events (OR $3 \cdot 23$ [95\% CI 1.37-7.69]; $\mathrm{p}=0 \cdot 007)$.

More adverse events took place in patients with Child-Pugh class B cirrhosis than those with ChildPugh A class cirrhosis (table 3), although the number of patients with Child-Pugh class B cirrhosis in this study was low.

\section{Discussion}

In this compassionate-use programme in patients with HCV genotype 1 or genotype 4 infection and cirrhosis, treatment with ombitasvir, paritaprevir, and ritonavir, with or without dasabuvir, plus ribavirin for 12 or 24 weeks resulted in a high proportion of patients achieving SVR12, similar to the proportions reported in clinical trials of these regimens. ${ }^{4-7}$ To our knowledge, this is the largest real-life study of ombitasvir, paritaprevir, and ritonavir, with or without dasabuvir, plus ribavirin in patients with cirrhosis. The concordance between the proportion of patients achieving a SVR12 in this study and in clinical trials provides reassurance that the reported efficacy of this treatment in clinical trials will translate to its use in routine clinical practice. The high proportion of patients achieving SVR12 in this study is similar to those reported in other real-life studies of ombitasvir, paritaprevir, and ritonavir, with dasabuvir, with or without ribavirin in patients with HCV genotype 1 infection and compensated cirrhosis. ${ }^{15-18}$

The strength of this study is that the study population was large and representative of patients with HCV genotype 1 infection with compensated cirrhosis in real-life clinical practice in Italy. This study has some limitations. Patients were not randomly assigned treatment regimens or durations, and the choice of treatment, patient visits, and laboratory testing were at the investigators' discretion. Moreover, the network of participating centres was established by AISF on the basis of the best expertise in HCV management, which could affect the external generalisability of our results. Furthermore, as efficacy and safety data were not provided by clinicians for all enrolled patients, it cannot be ruled out that the results of this study may slightly overestimate the efficacy and tolerability of ombitasvir, paritaprevir, and ritonavir, with or without dasabuvir, plus ribavirin.

At the time of this study, the label-recommended treatment of patients with genotype $1 \mathrm{~b}$ infection and cirrhosis was a 12 week regimen of ombitasvir, paritaprevir, ritonavir, with dasabuvir plus ribavirin. The label-recommended treatment of this group of patients has been updated in the European Union (EU) to a 12 week regimen of ombitasvir, paritaprevir, and ritonavir, with dasabuvir without ribavirin, based on the finding that $60(100 \%)$ of 60 patients with genotype $1 \mathrm{~b}$ infection and compensated cirrhosis achieved SVR12 with this regimen in the phase $3 \mathrm{~b}$ TURQUOISE-III trial. ${ }^{4}$

The proportion of patients achieving SVR12 did not differ by ribavirin dose, but SVR12 was achieved in more patients with early virological response versus those without. This difference in the proportion of patients achieving a SVR12 by timing of virological response was predominantly driven by the subgroup of patients with $\mathrm{HCV}$ genotype $1 \mathrm{~b}$ infection who were given ombitasvir, paritaprevir, and ritonavir, with dasabuvir plus ribavirin for 12 weeks, suggesting that longer duration treatment than 12 weeks might be 
beneficial in patients with genotype $1 \mathrm{~b}$ infection with late virological response. Despite these differences in the proportions of patients achieving a SVR12 between subgroups, the percentages were generally high (>90\%). In patients who achieved a SVR12, markers of liver function, including albumin and bilirubin, significantly improved at week 12 after treatment compared with baseline, suggesting that SVR12 is associated with improvement of liver function.

In this compassionate-use programme, ombitasvir, paritaprevir, and ritonavir, with or without dasabuvir, plus ribavirin was generally well tolerated, with few patients who discontinued treatment due to adverse events. Few patients had severe anaemia, and blood transfusion and erythropoietin use was low. The rate of hepatic decompensation in this study was low (four [1\%] of 734 patients), which is within the range for the previously reported annual risk of up to $6 \%$ for hepatic decompensation in patients with $\mathrm{HCV}$ infection and compensated cirrhosis. . $^{19,20}$

Increases in bilirubin concentrations reported in our study are consistent with those described in previous safety analyses of ombitasvir, paritaprevir, and ritonavir, with dasabuvir, with or without ribavirin. ${ }^{21}$ Increases in bilirubin concentrations reported in previous analyses were predominately indirect and related to the inhibition of the bilirubin transporters organic anion transporter polypeptide (OATP) 1B1 and 1B3 by paritaprevir and to ribavirin-induced haemolysis. ${ }^{21}$ These increases in bilirubin concentration generally resolved and were not associated with increases in serum ALT. ${ }^{21}$ Increases in bilirubin concentrations seen in our study are therefore probably due to inhibition of the bilirubin transporters OATP1B1 and 1B3 by paritaprevir and ribavirin-induced haemolysis, rather than hepatotoxicity caused by ombitasvir, paritaprevir, and ritonavir, plus dasabuvir treatment.

Estimated creatinine clearance decreased slightly during treatment with ombitasvir, paritaprevir, and ritonavir, with or without dasabuvir, plus ribavirin. This effect is consistent with that shown in patients with normal renal function (estimated glomerular filtration rate $[\mathrm{eGFR}]>90 \mathrm{~mL} / \mathrm{min}$ per $1.73 \mathrm{~m}^{2}$ ) in previous safety analyses of ombitasvir, paritaprevir, and ritonavir, with dasabuvir plus ribavirin in phase 2 and 3 clinical trials. ${ }^{22}$ Conversely, mean eGFR increased in patients with baseline renal impairment. ${ }^{22}$ There were too few patients with baseline renal impairment to establish whether the same effect was present in our study.

More adverse events occurred in patients with Child-Pugh B cirrhosis compared with those with Child-Pugh A cirrhosis, although the number of patients with Child-Pugh B cirrhosis in this study was low. At the time of this compassionate-use programme, other HCV treatment regimens without protease inhibitors were not available. After enrolment in this compassionate use programme was closed, guidance for the use of ombitasvir, paritaprevir, and ritonavir, with or without dasabuvir, plus ribavirin in patients with Child-Pugh B was changed, and ombitasvir, paritaprevir, and ritonavir, with dasabuvir, and ombitasvir, paritaprevir, and ritonavir are not recommended in patients with Child-Pugh B cirrhosis in the EU at present. ${ }^{2,3}$ Our data confirm the European Medicines Agency's recommendation not to use these regimens in patients with Child-Pugh B cirrhosis.

In conclusion, data collected in this compassionateuse programme suggest that the efficacy and safety profiles of ombitasvir, paritaprevir, and ritonavir, with or without dasabuvir, plus ribavirin in patients with HCV genotype 1 or 4 infection and advanced, compensated cirrhosis in a real-world setting are the same as those from clinical trials. Treatment of this population of patients is a high priority.

\section{Contributors}

All authors take full responsibility for the study design, data analysis and interpretation, and preparation of the manuscript; were involved in planning the analysis and drafting the manuscript; and approved the final draft manuscript.

\section{Declaration of interests}

SP has participated on advisory boards or has been a speaker for Gilead, AbbVie, Janssen, Bristol-Myers Squibb, MSD, and Roche. AAg reports grants from Gilead; and has participated on advisory boards and has been a speaker for Gilead, AbbVie, Janssen, MSD, and Bristol-Myers Squibb. AAl is a consultant for or has received research funding from Gilead, Roche, Bristol-Myers Squibb, Janssen, AbbVie, and MSD. AAn reports grants and personal fees from Gilead, Bristol-Myers Squibb, ViiV Healthcare, AbbVie, Janssen-Cilag, and Merck. RB has acted as an adviser or received speaker's fees from AbbVie, Gilead, Janssen-Cilag, MSD, and Roche. SB reports consulting fees from Gilead, Janssen, Roche, and MSD. AC has been a speaker or has participated on advisory boards for Gilead, AbbVie, Bristol-Myers Squibb, and MSD. GBG has been a speaker or adviser for AbbVie, Gilead, Janssen, Bristol-Myers Squibb, and MSD. EGG reports consulting fees from, research grants from, participation on advisory boards for, and is on the speaker's bureau for AbbVie, Bayer, Bristol-Myers Squibb, Gilead Sciences, GlaxoSmithKline, Janssen Cilag, MSD, and Novartis. VM is an adviser for AbbVie. CFP reports grants from, has been a speaker for, or has participated in advisory boards for Gilead, AbbVie, Janssen, Bristol-Myers Squibb, and MSD. MP reports grants from Gilead, ViiV Healthcare, and MSD; and personal fees for temporary scientific advice or talks in own events from AbbVie, Bristol-Myers Squibb, Roche, Roche Diagnostics, MSD, Gilead, and ViiV Healthcare. GR reports grants from Gilead, and MSD; and has been a speaker or participated in advisory boards for Gilead, AbbVie, Janssen, Bayer, Bristol-Myers Squibb, and MSD. MR has been a speaker or participated on advisory boards for Gilead, AbbVie, Bristol-Myers Squibb, and MSD. FCS has received grants, has been a speaker, or participated on advisory boards for Gilead, AbbVie, Janssen, Bristol-Myers Squibb, and MSD. EV reports participation on advisory committees or review panels for AbbVie, Bristol-Myers Squibb, Gilead, GlaxoSmithKline, MSD, and Roche; and has been a speaker and done teaching for AbbVie, GlaxoSmithKline, and Novartis. AC reports research grants, participation on advisory boards, and is a speaker for Gilead, AbbVie, Janssen, Bristol-Myers Squibb, and MSD. PR, SM, LP, $\mathrm{MMa}, \mathrm{AAs}, \mathrm{MMe}$, and ALZ declare no competing interests.

\section{Acknowledgments}

The views expressed in this article are the personal views of the authors and may not be understood or quoted as being made on behalf of or reflecting the position of the regulatory agencies or the committees or working parties of the regulatory agencies the authors work for. Medical writing support was provided by Andrew Kerr of Medical Expressions and funded by Dipartimento Biomedico di Medicina Interna e Specialistica dell'Universita di Palermo. 
References

1 European Association for the Study of the Liver. EASL recommendations on treatment of hepatitis C 2015. J Hepatol 2015; 63: 199-236.

2 AbbVie. Viekirax summary of product characteristics. 2016 https://www.medicines.org.uk/emc/medicine/29784 (accessed Aug 1, 2016).

3 AbbVie. Exviera summary of product characteristics. 2016. https://www.medicines.org.uk/emc/medicine/29785 (accessed Feb 15, 2016).

4 Feld JJ, Moreno C, Trinh R, et al. Sustained virologic response of $100 \%$ in HCV genotype $1 \mathrm{~b}$ patients with cirrhosis receiving ombitasvir/paritaprevir/r and dasabuvir for 12 weeks. J Hepatol 2016; 64: 301-07.

5 Poordad F, Hezode C, Trinh R, et al. ABT-450/r-ombitasvir and dasabuvir with ribavirin for hepatitis $\mathrm{C}$ with cirrhosis. $N$ Engl J Med 2014; 370: 1973-82

6 Waked I, Shiha G, Qaqish RB, et al. Ombitasvir, paritaprevir, and ritonavir plus ribavirin for chronic hepatitis $\mathrm{C}$ virus genotype 4 infection in Egyptian patients with or without compensated cirrhosis (AGATE-II): a multicentre, phase 3, partly randomised open-label trial. Lancet Gastroenterol Hepatol 2016; 1: 36-44.

7 Asselah T, Hezode C, Qaqish RB, et al. Ombitasvir, paritaprevir, and ritonavir plus ribavirin in adults with hepatitis $C$ virus genotype 4 infection and cirrhosis (AGATE-I): a multicentre, phase 3, randomised open-label trial. Lancet Gastroenterol Hepatol 2016; 1: 25-35.

8 Ferenci P, Bernstein D, Lalezari J, et al. ABT-450/r-ombitasvir and dasabuvir with or without ribavirin for HCV. N Engl J Med 2014; 370: 1983-92.

9 Zeuzem S, Jacobson IM, Baykal T, et al. Retreatment of HCV with ABT-450/r-ombitasvir and dasabuvir with ribavirin. N Engl J Med 2014; 370: 1604-14

10 Andreone P, Colombo MG, Enejosa JV, et al. ABT-450, ritonavir, ombitasvir, and dasabuvir achieves $97 \%$ and $100 \%$ sustained virologic response with or without ribavirin in treatment-experienced patients with HCV genotype 1b infection. Gastroenterology 2014; 147: 359-65.

11 Hezode C, Asselah T, Reddy K, et al. Ombitasvir plus paritaprevir plus ritonavir with or without ribavirin in treatment-naive and treatment-experienced patients with genotype 4 chronic hepatitis $\mathrm{C}$ virus infection (PEARL-I): a randomised, open-label trial. Lancet 2015; 385: 2502-09.

12 Feld JJ, Kowdley KV, Coakley E, et al. Treatment of HCV with ABT-450/r-ombitasvir and dasabuvir with ribavirin. $N$ Engl J Med 2014; 370: 1594-603.
13 Reau N, Poordad F, Enejosa J, et al. Preliminary safety and efficacy results from TOPAZ-II: a phase 3b study evaluating long-term clinical outcomes in HCV genotype 1-infected patients receiving ombitasvir/paritaprevir/r and dasabuvir +/- ribavirin. Hepatology 2015; 62: 732A.

14 Roche. Copegus EU summary of product characteristics. 2015. https://www.medicines.org.uk/emc/medicine/11755/SPC/ Copegus+200mg+Film-coated+Tablets/ (accessed Feb 15, 2016).

15 McCombs J, McGinnis J, Fox S, Tonnu-Mihara I. Analysis of the real-world effectiveness of direct acting antiviral treatments for hepatitis C in a large population. J Hepatol 2016; 64 (suppl 2): S217.

16 Calleja JL, Rincon D, Ruiz-Antoran B, Sacristan B. Effectiveness and safety of ombitasvir, paritaprevir, ritonavir and dasabuvir patients with genotype 1 chronic hepatitis $\mathrm{C}$ virus infection: results from the Spanish real world cohort. J Hepatol 2016; 64 (suppl 2): S218-19.

17 Zuckerman E, Ashkenasi E, Kovalev Y, Weitsman E. The real-world Israeli experience of treating chronic hepatitis $\mathrm{C}$, genotype 1 patients with advanced fibrosis with paritaprevir/ritonavir/ ombitasvir, dasabuvir with or without ribavirin: a large multi-center cohort. J Hepatol 2016; 64 (suppl 2): S137.

18 Hinrichsen H, Wedemeyer H, Christensen S, Sarrazin CM. Real-world safety and effectiveness of ombitasvir/ paritaprevir/r with dasabuvir and/or ribavirin in the German hepatitis $\mathrm{C}$ registry. J Hepatol 2016; 64 (suppl 2): S157.

19 Alazawi W, Cunningham M, Dearden J, Foster GR. Systematic review: outcome of compensated cirrhosis due to chronic hepatitis C infection. Aliment Pharmacol Ther 2010; 32: 344-55.

20 AASLD-IDSA Guidance Panel. HCV guidance: recommendations for testing, managing, and treating hepatitis C. Sept 16, 2016. http://hcvguidelines.org/ (accessed Oct 6, 2016).

21 AbbVie. Viekira Pak (ombitasvir, paritaprevir, and ritonavir tablets; dasabuvir tablets), co-packaged for oral use US prescribing Information. 2016. http://www.accessdata.fda.gov/drugsatfda_docs/ label/2014/206619lbl.pdf (accessed March 1, 2016).

22 Jacobson IM, Pockros PJ, Sulkowski MS, et al. Treatment with ombitasvir/ paritaprevir/r plus dasabuvir with ribavirin is not associated with adverse changes in renal function: an analysis of 4 phase 2/3 trials. GAJ 2015; 11: 65-66. 